\title{
Disparity Sensitivity of Frontal Eye Field Neurons
}

\author{
STEFANO FERRAINA, MARTIN PARÉ, AND ROBERT H. WURTZ \\ Laboratory of Sensorimotor Research, National Eye Institute, National Institutes of Health, Bethesda, Maryland 20892
}

Ferraina, Stefano, Martin Paré, and Robert H. Wurtz. Disparity sensitivity of frontal eye field neurons. J. Neurophysiol. 83: 625-629, 2000. Information about depth is necessary to generate saccades to visual stimuli located in three-dimensional space. To determine whether monkey frontal eye field (FEF) neurons play a role in the visuo-motor processes underlying this behavior, we studied their visual responses to stimuli at different disparities. Disparity sensitivity was tested from $3^{\circ}$ of crossed disparity (near) to $3^{\circ}$ degrees of uncrossed disparity (far). The responses of about two thirds of FEF visual and visuo-movement neurons were sensitive to disparity and showed a broad tuning in depth for near or far disparities. Early phasic and late tonic visual responses often displayed different disparity sensitivity. These findings provide evidence of depth-related signals in FEF and suggest a role for FEF in the control of disconjugate as well as conjugate eye movements.

\section{IN T R O D UCT ION}

Eye movements permit primates to explore their visual environment. Conjugate movements that move the two eyes equally bring the visual axes to a new point at the same depth of fixation, but disconjugate movements are necessary every time the visual images are at different depths. These two types of eye movements are controlled by the saccadic and vergence systems that normally interact to subserve binocular vision, as demonstrated by recent behavioral studies (Chaturvedi and van Gisbergen 1998; Erkelens et al. 1989; Maxwell and King 1992; Zee et al. 1992).

The neurophysiology of the conjugate saccadic system in both cortical and subcortical structures has been studied extensively (for reviews see Wurtz and Goldberg 1989). However, less is known about the neural system involved in the generation of disconjugate eye movements. Some important vergence centers have been identified in the midbrain (Judge and Cumming 1986; Mays 1984; for a review see Mays and Gamlin 1995), but there is scant knowledge about cortical areas playing a definitive role in the control of disconjugate eye movements. One hypothesis, not fully tested, is that some of the cortical areas known to be involved in the generation of conjugate saccades also play a role in the production of disconjugate eye movements.

Depth discrimination is a prerequisite for correct foveation of visual stimuli located in three-dimensional space. The most important depth cue is retinal image disparity, which results when visual images at different depths in the field impinge onto the two retinas at different positions (Fig. 1A). Among the neural structures forming the interface between the visual and saccadic systems, the lateral intraparietal (LIP) area has been

The costs of publication of this article were defrayed in part by the payment of page charges. The article must therefore be hereby marked "advertisement" in accordance with 18 U.S.C. Section 1734 solely to indicate this fact. shown to contain disparity-sensitive neurons (Gnadt and Mays 1995). Moreover, this depth-related signal is carried by LIP output neurons projecting to two other major visuo-saccadic centers, the midbrain superior colliculus (SC) (Gnadt and Beyer 1998) and the frontal eye field (FEF) in frontal cortex (Ferraina et al. 1999). Further preliminary data suggest that the FEF may also be involved in the control of disconjugate eye movements. First, Jampel (1960) reported that stimulation of the frontal lobe could elicit both saccadic and vergence eye movements. Second, Gamlin et al. (1996) identified neurons in a prearcuate area that displayed activity related to tracking in depth. Given these data and the strong anatomic connections between area LIP and FEF (for a review see Schall 1997), we investigated whether the visual activation of FEF neurons is tuned in depth and thereby possibly involved in the visuomotor processes underlying disconjugate eye movements.

\section{METHODS}

Two male monkeys (Macaca mulatta) were prepared for chronic recording of single-neuron activity and of the positions of both eyes in a single surgical procedure described previously (Munoz and Wurtz 1993). All animal care and experimental procedures were approved by the Institute Animal Care and Use Committee and complied with Public Health Service Policy on the humane care and use of laboratory animals.

The anterior bank of the arcuate sulcus was delimited with the help of magnetic resonance imaging (MRI) of the monkey's brain. The exact location of FEF was identified physiologically by the concentration of neurons with visual and saccade-related activities and by the ability to evoke saccades with $<50 \mu \mathrm{A}$ current.

All tasks were performed in dim ambient light with visual stimuli (single dots, $0.25^{\circ}$ diam) generated by a video projector and backprojected onto a translucent tangent screen positioned $57 \mathrm{~cm}$ in front of the head-restrained monkey. First, we determined the location of the receptive field and the discharge properties of each isolated neuron using visual and memory delayed saccade tasks (Paré and Wurtz 1997). Each trial started with the appearance of a central visual fixation point and after $500-800 \mathrm{~ms}$ of fixation an eccentric visual target appeared and remained present either for $100 \mathrm{~ms}$ in the memory trials or throughout the visual trials. After a 500-1000 ms delay period, the fixation point disappeared signaling the monkey to make a saccade to either the remembered location of the target or the still visible target. Following this classification, the disparity sensitivity of each neuron was tested using a visual fixation task. To produce disparity stimuli, we divided the tangent screen into two halves and projected a fixation point and an eccentric visual stimulus on each half. The visual stimulus appeared after 500-800 ms of fixation and remained present for $1000 \mathrm{~ms}$. The location of the stimulus in the visual field was the same that elicited optimal activation in the delayed saccade tasks but, this time, maintained fixation was required. A base-cut prism positioned in front of each eye deflected the line of sight of the right eye to the right, and that of the left eye to the left (Eifuku and Wurtz 1999). Retinal disparity was induced by changing 

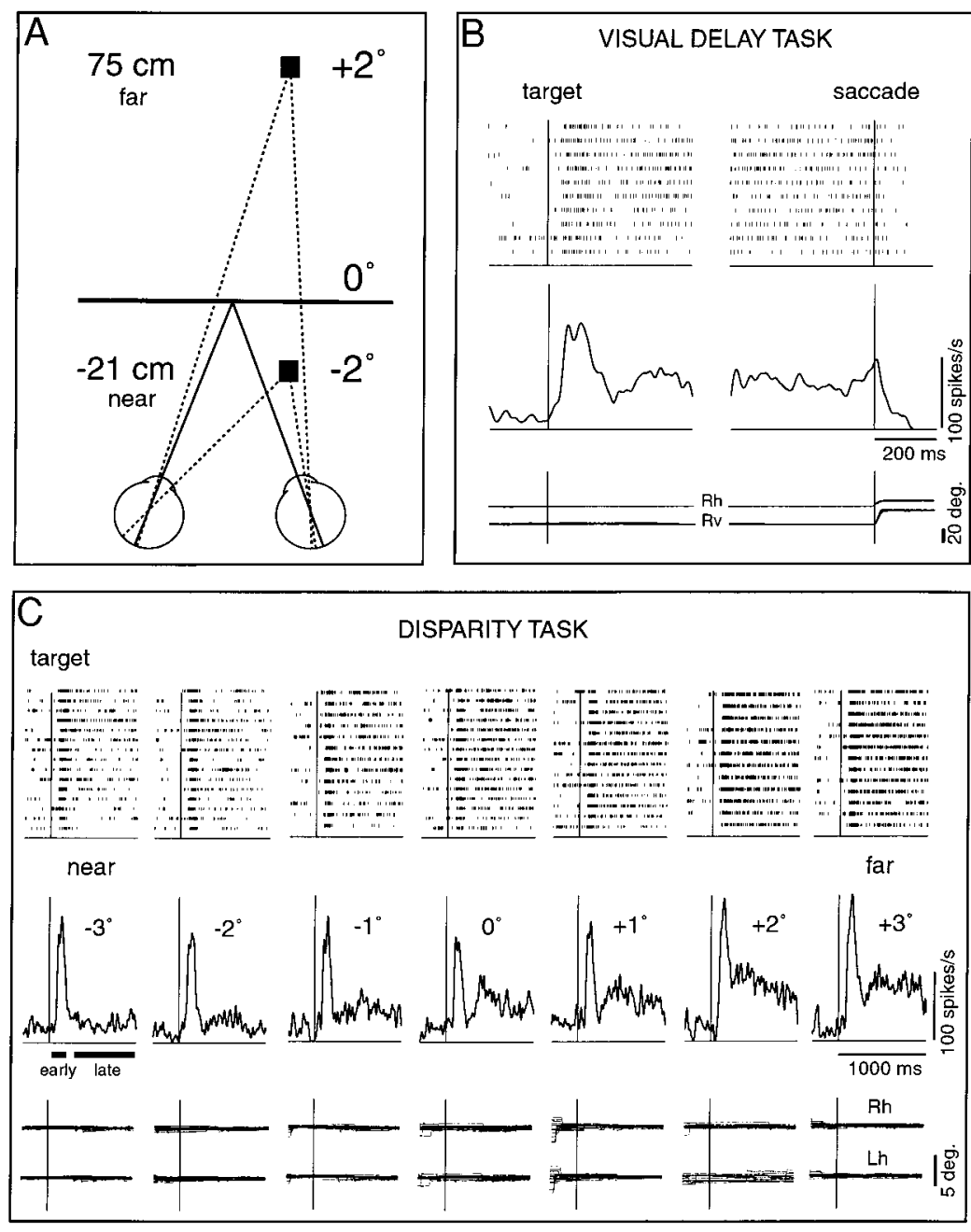

FIG. 1. Determination of disparity sensitivity of frontal eye field (FEF) neurons. A: schematic of near and far disparity. $\mathbf{\square}$, target displacement with disparity of +2 or -2 degrees. Numbers on the left side indicate relative perceived location with respect to the fixation plane (see METHODS). - , line of sight when the monkey fixates the central target; - - -, retinal projections of the 2 targets. $B$ : neuronal activity and spike density function of the average firing rate (Gaussian pulse of $10 \mathrm{~ms}$ ) of a neuron in the visual delayed saccade task. Bottom: eye traces (Rh, right eye horizontal component; Rv, right eye vertical component). Left of the figure is aligned on the target presentation and the right on the saccade beginning. Visual target was present from its appearance throughout the trial. $C$ : neuronal activity and spike density function for the same neuron in $B$ tested in the disparity task. Bottom: horizontal components from both eyes ( $\mathrm{Rh}$ and Lh). Analysis epochs are indicated as such: early correspond to the early visual activation and late to the late visual activation. the relative position of the visual stimuli displayed on the half screens and, to survey a broad range of disparity, it varied from -3 to $+3^{\circ}$ in steps of $1^{\circ}$. Different disparity values were presented in a random order. The fixation point was always on the screen at $0^{\circ}$ disparity (Fig. $1 A$ ). With a $57-\mathrm{cm}$ screen distance and an interocular distance of 3.5 $\mathrm{cm}$, the uncrossed disparity stimuli of $+1,+2$, and $+3^{\circ}$ appeared to be located, respectively, at $22.7,75.2$, and $330.8 \mathrm{~cm}$ beyond the plane of the screen (far). Crossed disparity of $-1,-2$, and $-3^{\circ}$ created a binocular stimulus located 12.6, 20.7, and $26.3 \mathrm{~cm}$ in front of the plane of the screen (near). Throughout the trial, the monkey maintained fixation with both eyes within a computer-controlled window of $\pm 1-1.5^{\circ}$. Adequate fusion of the fixation stimuli was inferred by the consistency from trial to trial of the position of both eyes within the electronic window.

\section{RES U L T S}

Using the data collected in the visual and memory delayed saccade tasks, we separated our sample of 57 FEF neurons (36 from monkey $\mathrm{C}$ and 21 from monkey $\mathrm{A}$ ) into different groups with a classification scheme similar to that used in previous descriptions of FEF neurons (Bruce and Goldberg 1985; Segraves and Goldberg 1987). Most neurons $(n=35)$ displayed a target-locked visual response that usually continued throughout the delay epoch and a presaccadic increase of activity in either visual or memory trials; these were classified as visuomovement neurons (Fig. $1 B$ ). In contrast, the activity of visual neurons did not show a presaccadic increase $(n=11)$, whereas movement neurons had only saccade-related discharges $(n=$ 11). None of the 11 movement neurons showed an emergent visual response for any of the disparity values tested. We studied the disparity sensitivity of the 46 neurons that had visual responses. All the neurons had a large receptive field centered in the contralateral visual field. Because of the optics of the prisms and the display arrangement in the fixation task we were limited to testing visual receptive fields with eccentricities $<15^{\circ}$. Of these, seven were judged online to show no modulation with disparity changes. The remaining 39 neurons were examined quantitatively.

Figure $1 C$ shows the responses of a typical visuo-movement neuron (same as Fig. 1B) in the disparity task. Neuronal activation following stimulus presentation consisted of an early phasic response and a later tonic response. Both responses with the far stimuli were stronger than with the near stimuli. However the changes in the two responses associated with the change in disparity were not identical. To quantify this neuronal activity, we measured the discharge rate during an early visual epoch lasting 50-200 ms after target onset and a late visual epoch 300-1000 ms after target onset and compared them across different disparities. In the example of Fig. $1 C$, both the early and the late visual responses were significantly different during at least one disparity value [Kruskal-Wallis 
A

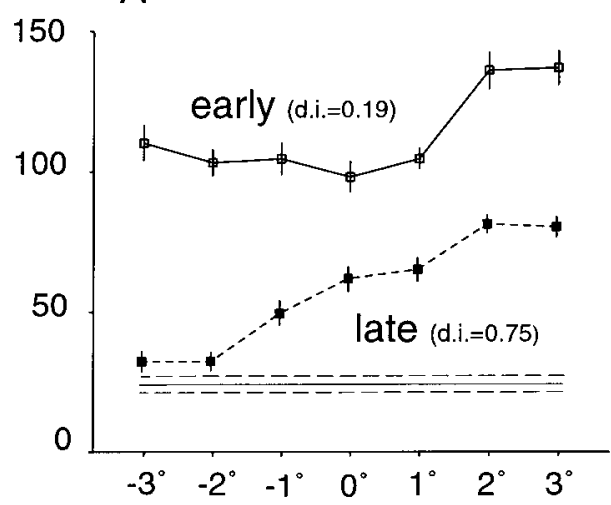

B
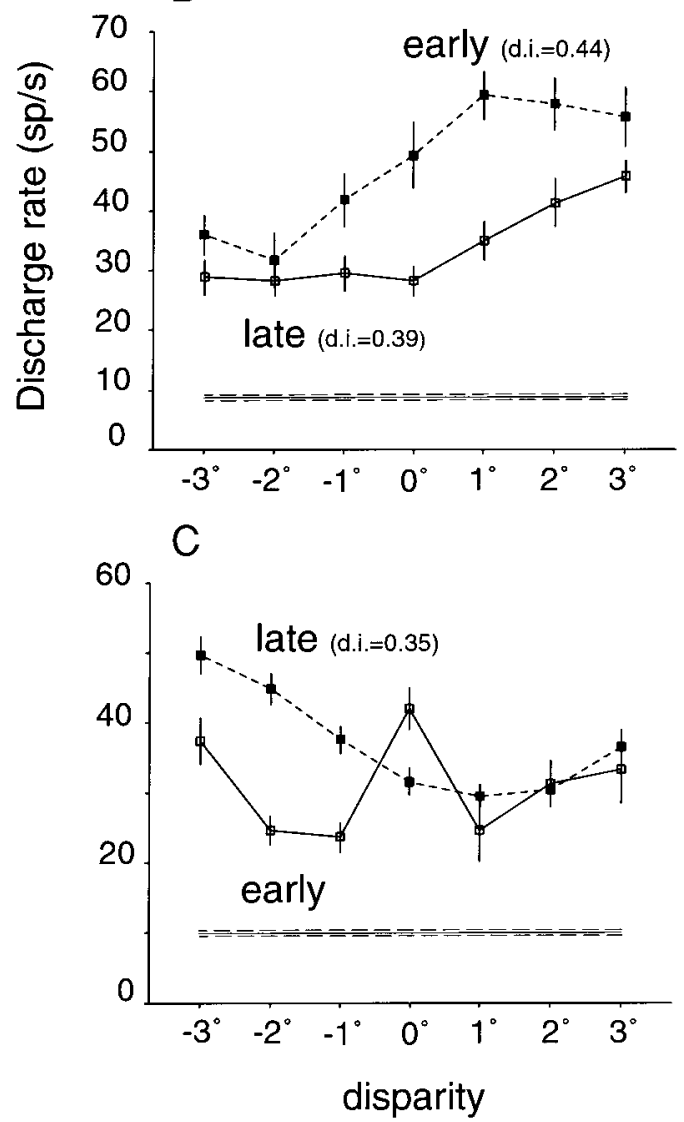

FIG. 2. Disparity tuning of FEF neurons. Examples from 3 neurons. Mean and SE discharge rate are shown at each disparity value tested. $\square$, activity relative to the early visual response; $\mathbf{n}$, activity relative to the late visual response. Bottom lines indicate mean and $\mathrm{SE}$ of the control activity. Figure $2 A$ shows the same neuron as in Fig. 1, $B$ and $C$. Disparity indices (d.i.) are shown for each curve.

analysis of variance (ANOVA) on ranks, $P<0.05]$. Across our sample of visually responsive neurons, the activity of $80 \%$ of the neurons (31/39) was significantly modulated by changes in retinal disparity during at least one of the two analysis epochs; 46\% (18/39) during both epochs. If we took into account the seven neurons that were qualitatively judged to be nonmodulated, about two-thirds (31/46) of the visually responsive neurons could be considered sensitive to disparity.

The lower traces in Fig. $1 C$ show horizontal positions for the two eyes during fixation of the stimulus at different disparities.
We saw no evidence of systematic changes in the position of the two eyes, either on successive trials or between the early and late visual epochs of the trials. In other words, the visual stimulation never elicited minute vergence eye movements.

Figure 2 illustrates the variation in the disparity tuning for both the early and the late visual epochs. The example neurons in Fig. $2 A$ (same neuron as in Fig. $1, B$ and $C$ ) and $2 B$ showed broadly tuned responses that were strongest with far disparities. In addition, both neurons displayed early and late responses that were different, although the responses maintained the same depth preference (far) even when the optimal disparity values differed (Fig. 2B). This similarity in depth preference (near or far) was true for all but three of the neurons studied (36/39). Each of these latter three neurons had a sharp tuning curve centered at zero disparity in one of the two analysis epochs. Figure $2 C$ illustrates one such neuron.

To estimate the strength of the depth tuning, we first computed the mean discharge rate for the three far disparities and for the three near disparities. Then, using these two rate values, we calculated the disparity index previously employed by Roy et al. (1992): Disparity Index $=1-(N-C) /(P-C)$ where $P$ (preferred) is the highest rate obtained for either the near or the far disparities, $N$ (nonpreferred) is the lowest rate value obtained, and $C$ (control) is the average discharge rate during the final 300-ms fixation before target onset. The larger the disparity index, the larger the difference in the response to near and far disparities. For example, the disparity indices for the neuron in Fig. $2 A$ for the early and late visual epochs were 0.19 and 0.75 , respectively.

Figure 3 shows the range of values of the index for the early and late visual epochs and the relation between them for all neurons examined (excluding the three neurons with a sharp zero tuned response). Ninety-seven percent (35/36) of the neurons showed a disparity index $>0.20$ (i.e., $>20 \%$ change in activity) during at least one of the two analysis epochs. The mean disparity index for the early and the late visual epochs was 0.44 and 0.60 , respectively; not a statistically significant difference (paired $t$-test; $P=0.14$ ). However, there frequently

\section{DISPARITY INDEX}

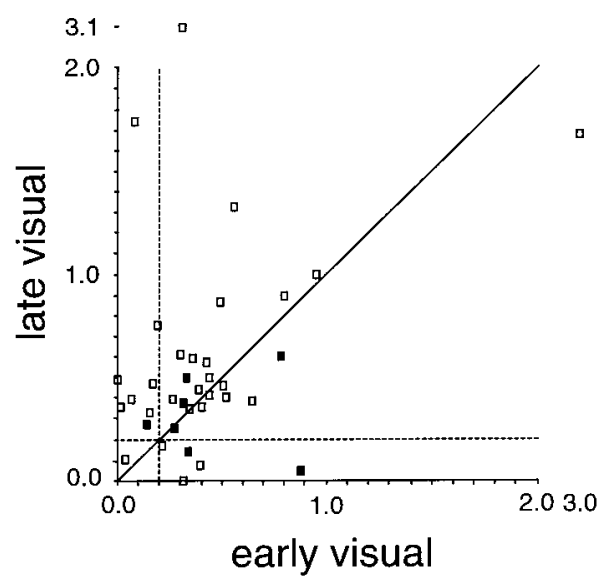

FIG. 3. Scatter plot of the disparity index for the early and late visual responses. - , equality line; - - -, levels of 0.2 for the disparity index. Seventyfive percent (27/36) of the neurons showed a disparity index $>0.20$ during the early visual epoch, 83\% (30/36) during the late visual epoch, and 58\% (21/36) in both epochs. $\square$, visuo-movement neurons; $\mathbf{m}$, visual neurons. Both axes are interrupted to include the 2 large values. 
was a substantial difference between the early and the late indices of individual neurons. Of all the neurons with disparity indices $>0.2$, most displayed a broad tuning and could qualitatively be classified as either far (e.g., Fig. 2, $A$ and $B$ ) or near (e.g., Fig. $2 C, L V$ epoch) neurons (Poggio and Fisher 1977). In our sample, neurons tuned for far disparities predominated ( $70 \%$ of both the early and the late visual responses).

\section{I S C U S S I O N}

We found that many visually responsive FEF neurons were sensitive to retinal disparity. Most of them were broadly tuned in depth, and the disparity tuning of the early and late visual activation often exhibited different profiles.

Neurons showing disparity sensitivity have been found in the primary visual area (V1) (Poggio and Fisher 1977) and in many other extrastriate visual areas, including the middle temporal (MT) area (Maunsell and Van Essen 1983) and the medial superior temporal (MST) area (Eifuku and Wurtz 1999; Roy et al. 1992) (for a review see Poggio 1995). In addition, neurons in parietal area LIP are tuned in depth (Gnadt and Mays 1995). Because FEF is anatomicly connected with MT, MST, and LIP (Schall et al. 1995), it is not surprising to find a depth signal in FEF neurons as well. Nevertheless, the FEF is the first frontal motor area in which such a visual property has been demonstrated.

The existence of depth-related signals indicates a possible function for FEF in shifting fixation to visual stimuli located in three-dimensional space. The predominance of broadly tuned neurons suggests a role in the processing of coarse disparity (Bishop and Henry 1971), which could be important for initiation of vergence movements because it carries information about the target distance relative to the fixation point (Poggio and Fisher 1977). Fine disparity, in addition to its role in stereoscopic perception, may instead be involved in guiding the completion of vergence and the maintenance of binocular fixation (Poggio 1995). In addition to disparity, representation of depth could be extracted from other physical parameters of the visual stimulus (e.g., size, blur). FEF neurons could thus be generally sensitive to depth just as has been shown for antecedent cortical areas (Gnadt and Mays 1995; Sakata et al. 1985; Zeki 1974), a hypothesis that remains to be tested.

The depth tuning of the early phasic and late tonic response was frequently different. This discrepancy could simply be explained by the fixation task not engaging optimally the visuo-motor functions of FEF neurons. Alternatively, it could potentially indicate a differential participation of these two phases in the visuo-motor process for the generation of disconjugate movements. Perhaps the phasic response is more related to the localization of the saccadic goal in three-dimensional space, whereas the tonic activation is more related to the later processes leading to disconjugate movement execution. Information about the disparity tuning of the movement-related activity may provide further support for this hypothesis, but it is not available from this study because of limitations in the method used for disparity testing.

Our results in FEF along with those obtained in area LIP (Gnadt and Mays 1995) suggest that these two major cortical areas involved in saccade production probably have a role in the guidance of disconjugate eye movements. Moreover, just as the saccade-related neurons in the SC intermediate layers may receive a depth-related signal from LIP (Gnadt and Beyer 1998), the same signal could be provided by visually responsive FEF neurons, some of which project to the SC (Segraves and Goldberg 1987; Sommer and Wurtz 1999). The identification, in a complementary study (Ferraina et al. 1999), of one FEF disparity sensitive neuron as an SC projection neuron, indicates that this is indeed likely. Altogether, these data strongly imply that the SC could be involved in the generation of disconjugate movements (see also Chaturvedi and van Gisbergen 1999). Furthermore, since LIP neurons projecting to FEF also are sensitive to stimulus disparity (Ferraina et al. 1999), LIP, FEF, and SC probably form a network for the control of disconjugate as well as conjugate eye movements.

We thank the Laboratory of Diagnostic Radiology Research for magnetic resonance images.

S. Ferraina was supported by a Human Frontier Science Program Organization fellowship.

Address for reprint requests: S. Ferraina, Laboratory of Sensorimotor Research, NEI/NIH, 9000 Rockville Pike, Bldg. 49, Room 2A50, Bethesda, MD 20892-4435.

Received 27 August 1999; accepted in final form 24 September 1999.

\section{REFERENCES}

Bishop, P. O. And Henry, G. H. Spatial vision. Annu. Rev. Psychol. 22: 119-161, 1971.

Bruce, C. J. ANd Goldberg, M. E. Primate frontal eye fields. I. Single neurons discharging before saccades. J. Neurophysiol. 53: 603-635, 1985.

Chaturvedi, V. AND van Gisbergen, J.A.M. Shared target selection for combined version-vergence eye movements. J. Neurophysiol. 80: 849-862, 1998.

Chaturvedi, V. And van Gisbergen, J.A.M. Perturbation of combined saccade-vergence movements by microstimulation in monkey superior colliculus. J. Neurophysiol. 81: 2279-2296, 1999.

EIFUKU, S. AND WURTZ, R. H. Response to motion in extrastriate area MSTl: Disparity sensitivity. J. Neurophysiol. 82: 2462-2475, 1999.

ERKelens, C. J., SteinMan, R. M., AND Collewisn, H. Ocular vergence under natural conditions. Continuous changes of target distance along the medial plane. Proc. R. Soc. Lond. B. Biol. Sci. 236: 417-440, 1989.

Ferraina, S., ParÉ, M., AND WuRTZ, R. H. Discharge properties of lateral intraparietal neurons antidromically activated by frontal eye field stimulation. Soc. Neurosci. Abstr. 25: 806, 1999.

GAMLIN, P. D., YoON. K., AND ZhANG, H. The role of cerebro-ponto-cerebellar pathways in the control of vergence eye movements. Eye 10: 167-171, 1996.

GNADT, J. W. AND MAYs, L. E. Neurons in monkey parietal LIP are tuned for eye-movement parameters in three-dimensional space. J. Neurophysiol. 73: 280-297, 1995

GNADT, J. W. AND BEYER, J. Eye movements in depth: what does the monkey's parietal cortex tell the superior colliculus? Neuroreport 9: 233-238, 1998.

JAMPEL, R. S. Convergence, divergence, pupillary reactions and accommodation of the eyes from faradic stimulation of the macaque brain. J. Comp. Neurol. 115: 371-399, 1960.

JudGe, S. J. AND Cumming, B. G. Neurons in the monkey midbrain with activity related to vergence eye movement and accommodation. J. Neurophysiol. 55: 915-930, 1986.

Maunsell, H. R. And van Essen, D. C. Functional properties of neurons in middle temporal visual area of the macaque monkey. II. Binocular interactions and sensitivity to binocular disparity. J. Neurophysiol. 49: 1148-1167, 1983.

MAYS, L. E. Neural control of vergence eye movements: convergence and divergence neurons in the midbrain. J. Neurophysiol. 51: 1091-1108, 1984.

Mays, L. E. AND Gamlin, P. D. Neuronal circuitry controlling the near response. Curr. Opin. Neurobiol. 5: 763-768, 1995.

MAXWELL, J. S. AND KING, W. M. Dynamics and efficacy of saccade-facilitated vergence eye movements in monkeys. J. Neurophysiol. 68: 1248-1260, 1992.

MunOZ, D. P. AND WuRTZ, R. H. Fixation cells in monkey superior colliculus. I. Characteristics of cell discharge. J. Neurophysiol. 70: 559-575, 1993. 
Paré, M. And Wurtz, R. H. Monkey posterior parietal cortex neurons antidromically activated from superior colliculus. J. Neurophysiol. 78: $3493-$ 3497, 1997.

Poggio, G. F. Mechanisms of stereopsis in monkey visual cortex. Cereb. Cortex 5: 193-204, 1995.

PogGio, G. F. AND Fisher, B. Binocular interaction and depth sensitivity in striate and prestriate cortex of behaving rhesus monkey. J. Neurophysiol. 40: 1392-1405, 1977.

Roy, J. P., Komatsu, H., AND WuRTZ, R. H. Disparity sensitivity of neurons in monkey extrastriate area MST. J. Neurosci. 12: 2478-2492, 1992.

Sakata, H., Shibutani, H., Kawano, K., and Harrington, T. L. Neural mechanisms of space vision in the parietal association cortex of the monkey. Vision Res. 25: 453-463, 1985.

Schall, J. D., Morel, A., King, D. J., ANd Bullier, J. Topography of visual cortex connections with frontal eye field in macaque: convergence and segregation of processing streams. J. Neurosci. 15: 4464-4487, 1995.
Schall, J. D. Visuomotor areas of the frontal lobe. In: Extrastriate Cortex of Primates. Cerebral Cortex, edited by K. Rockland, A. Peters, and J. Kaas. New York: Plenum, 1997, vol. 12, p. 527-638.

Segradies, M. A. AND Goldberg, M. E. Functional properties of corticotectal neurons in the monkey's frontal eye field. J. Neurophysiol. 58: 1387-419, 1987.

SOMMER, M. A. AND WuRTZ, R. H. The topographic organization of signals sent from frontal eye field to the superior colliculus. Soc. Neurosci. Abstr. 25: 805, 1999.

Wurtz, R. H. And GoldberG, M. E. The Neurobiology of Saccadic Eye Movements, Reviews of Oculomotor Research, Vol. III. Amsterdam: Elsevier, 1989

Zee, D. S., FitzGibbon, E. J., And Optican, L. M. Saccade-vergence interactions in humans. J. Neurophysiol. 68: 1624-1641, 1992.

ZeKI, S. M. Cell responding to changing image size and disparity in the cortex of the rhesus monkey. J. Physiol. (Lond.) 242: 827-841, 1974. 